\title{
PIETRO PAOLO MORRA
}

Il 17 aprile scorso spirava in Torino il dott. ing. Pietro Paolo Morra, professore di fisica tecnica al R. Museo Industriale, e di fisica generale alla R. Accademia Militare ed alla R. Scuola di Guerra.

La vita e l'opera scientifico-didattica del Morra intimamente si collegano a quelle di due illustri fisici - Galileo Ferraris e Giuseppe Basso, - che ebbero per il Morra grande stima ed affetto, cosi da sceglierlo a loro collaboratore. A questi suoi maestri venerati il Morra portava pari riconoscenza: unendoli in un medesimo sentimento di reverenza e di rimpianto, egli amava ricordare non solo l'intima amicizia dei due grandi ingegni, ma ancora le dotte discussioni fra i due scienziati, alle quali egli ebbe la ventura di assistere.

Di Giuseppe Basso il Morra fu assistente prima alla R. Università di Torino nel periodo dal 1876 al 1878, nel quale detto professore vi tenne la cattedra di fisica sperimentale, e poi alla R. Accademia Militare: in quest' ultima cattedra, in seguito a concorso, fu chiamato a succedergli nel 1885.

Assunto sin dal 1879 da Galileo Ferraris ad assisterlo nel corso di fisica tecnica presso il R. Museo Industriale, il Morra, ammirato dalle splendide doti non solo dell'alta mente, ma ancora del gran cuore del sommo scienziato, portò a lui un affetto fraterno, e gli fu sino alla morte compagno fedele e devoto, amico affezionato; premuroso sempre di sollevarne le fatiche, perchè maggior tempo potesse dedicare ai suoi studi prediletti, nulla cercò per sè, pago del dovere compiuto e della stima e dell'affetto grande che a lui di tutto cuore contraccambiava il Ferraris. In molteplici occasioni Ga- 
lileo Ferraris ebbe a testimoniare in quale alta considerazione tenesse il suo assistente: cosi, quando nello stesso anno 1879 venivano creati presso il $R$. Museo Industriale i corsi speciali di industrie meccaniche e chimiche, volle che il corso di fisica generale ed applicata fosse a lui affidato: cosi ancora quando nel 1886 istitui il corso superiore di Elettrotecnica, ottenne che il Morra fosse chiamato a coadiuvarlo in quello di fisica tecnica, ed anzi d'allora in poi gli lasciò ben spesso il compito di svolgere completo questo corso: da ultimo il Ferraris, chiamato alla dignita senatoriale, prevedendo che anche maggior lavoro arrebbe dovuto lasciare al Morra, divisava, come ebbe a dichiarare ai suoi amici, un riordinamento degli insegnamenti, per il quale fosse dato a questi l'onore della cattedra, come già ne sopportava tutte le fatiche: solo la morte prematura che ne troncò la preziosa esistenza, gli impedi di tradurre in atto il suo divisamento.

Alla morte di Galileo Ferraris il Morra fu subito chiamato a succedergli nella cattedra alla $R$. Scuola di Guerra, e più tardi nel 1901 anche in quella al R. Museo Industriale.

Pietro Paolo Morra fu essenzialmente ed anzitutto un grande studioso: sin dai suoi anni giovanili, quando, ottenuta nel 1870 la Iaurea in fisico-matematica presso la $R$. Università di Torino, le impellenti necessità della vita lo costringevano a dedicare molta parte dell'opera sua nell'insegnamento dell' aritmetica nei ginnasii, mentre pur continuava gli studii di ingegneria presso la Scuola di Applicazione, egli veniva preparando la sua mente collo studio profondo e minuto delle opere classiche dei maestri della fisica: in tale studio continuò per tutta la vita; anche nei suoi ultimi anni, quando la molteplicità dei corsi lo oberava di lavoro, malgrado la sua salute già malferma, egli trovava tempo di leggere, analizzare e discutere non solo le opere principali che sulla fisica generale ed applicata si venivano man mano pubblicando, ma ancora le memorie singole che comparivano negli Atti delle Accademie e sui giornali tecnici. Le principali nuove espe- 
rienze egli amava riprodurre pur coi pochi mezzi di cui disponeva in quel suo piccolo laboratorio speciale che con tanto amore si era venuto formando, nel quale io amo rafigurarmelo intento allo stıdio ed alla preparazione delle sue lezioni sperimentali; e voglio ancora qui ricordare il dolore profondo che egli provò, quando anni fa, per l'ampliamento dell' Istituto, il piccone demolitore ebbe ad abbattere ciò che gli era tanto caro, senza che venisse al Laboratorio suo data in allora nuova opportuna sede.

Con questo studio continuo, minuto e diligente, il Morra si era venuto formando una coltura scientifica vasta e profonda. Questo studio fu l' unico srago e diletto della sua vita, e valse pure in un coll'affetto grande della sua gentile e valorosa compagna e di quell'amore della sua bimba a confortarlo nei dolori e dispiaceri che non gli furono purtroppo risparmiati nella sua carriera.

L' esempio dei maestri sommi alla scuola dei quali egli si era venuto educando, la coltura profonda che, come ho dianzi ricordato, egli aveva col diuturno studio acquistato, erano certo la preparazione migliore per formare in lui il provetto professore. L'insegnamento egli fece scopo e fine della sua esistenza; all' insegnamento della fisica nei varii corsi che furono a lui affidati, egli dedicò ogni sua attività.

Nelle due Scuole Superiori militari, ove l' insegnamento della fisica nun deve proporsi scopi particolari di applicazione ma essere esclusivamente diretto a dare ai giovani ufficiali anche in questo ramo della scienza la necessaria coltura, il prof. Morra seppe mantenere nel suo corso un giusto equilibrio fra la parte teorica e quella sperimentale: nelle tratta. zioni teoriche, senza venir meno al rigore scientifico, egli si fece uno studio costante di scegliere i mezzi di analisi più semplici e perció tanto più facilmente comprensibili : le numerose esperienze poi che egli veniva man mano riproducendo nel corso, mentre tenevano desta $l^{\prime}$ attenzione degli uditori, erano da lui prescelte per modo non solo da far ri- 
saltare evidente il comportamento dei varii fenomeni e la deduzione delle leggi che li regolano, ma ancora da porre in rilievo i principii su cui si fondano le applicazioni, che per i continui progressi della scienza e della tecnica, tali fenomeni ricevono nella vita pratica.

$\mathrm{Nel}$ corso di fisica tecnica, al quale, se possibile, portò anche maggiore cura ed amore, il prof. Morra non dimenticò mai che per l' ingegnere la matematica e la scienza non sono fine a sè stesse, ma unicamente mezzo per la migliore soluzione dei problemi della tecnica: perciò egli, che pure era profondo conoscitore delle discipline matematiche, rifuggi sempre da lunghe trattazioni analitiche, le quali, per quanto eleganti, riescono inutili all' ingegnere quando non approdino ad un pratico risultato. Questo era di regola il suo metodo di insegnamento: posto dapprima il problema in tutta la sua generalità, vi introduceva subito le ipotesi semplificative atte a facilitarne la soluzione; discuteva poi il valore di queste ipotesi, e ne deduceva quindi la portata delle formole risolutive e l'applicazione che possono ricevere nella tecnica; in questi ragionamenti sapeva trarre ampio partito dai risultati delle esperienze dirette, dei quali faceva abbondanti citazioni: da ultimo per meglio dimostrare la pratica applicazione delle formole egli non disdegnava di softermarsi a svolgere esempi numerici di calcolo diretto. In sostanza il Morra, come egli stesso ebbe già a dire del suo grande Maestro, aveva di mira di formare negli allievi quel buon senso pratico, che deve essere di guida nella soluzione dei problemi di ingegneria, difficili sempre, assai spesso indeterminati.

Ad un altro corso il prof. Morra come ho gia ricordato dedicò ancora la sua attività, quello di fisica generale ed applicata per i corsi speciali di Industrie chimiche e meccaniche presso il R. Museo Industriale. Nella istituzione affrettata di questi corsi era mancata una chiara visione di ciò che si volesse ottenere, talchè si diedero alla scuola due scopi in gran parte discordanti; preparare dei direttori tecnici per le varie industrie e formare dei professori per le scuole di arti e mestieri. Questa discordanza di intenti, mentre fu cagione prima della vita poco florida che queste scuole hanno sinora trascinato, 
non poteva se non influire dannosamente sullo sviluppo degli stessi corsi: il prof. Morra seppe però nel suo insegnamento compenetrare abbastanza bene questi intenti disparati: difatti, mentre nella trattazione generale, riprendendo da principio i varii rami della fisica, partiva da esperienze semplici e facili, per assurgere poi ai fenomeni complessi, alle leggi che li regolano, alla intima correlazione fra fenomeno e fenomeno, istradando cosi gli allievi a quel metodo induttivo col quale deve essere impartito l' insegnamento ai giovani operai non solo digiuni di ogni cognizione scientifica, ma ben spesso anche ignari di ogni metodo: invece nello studio delle applicazioni si soffermava a descrivere minutamente le parti costitutive fondamentali dei varii apparecchi tecnici, e ne spiegava le proprieta ed il comportamento, per modo da addestrare gli allievi a saperne guidare e dirigere il funzionamento e la manutenzione.

Di questo corso egli si occupó con vero intelletto di amore e con vivo rincrescimento lo abbandonó quando fu chiamato a più importanti insegnamenti.

Il prof. Morra non fu nè oratore nè dicitore brillante, ebbe però parola facile ed elegante, colla quale sapeva, in quelle lezioni dense sempre di pensiero e di concetto, che egli con tanto studio ed amore veniva preparando, tener desta l'attenzione degli allievi, che, mentre ammiravano in lui la profonda dottrina, ne stimavano il carattere integro di specchiata rettitudine, ne amavano l'animo dolce e mite.

Un professore di scienza applicata non puó formarsi esclusivamente sullo studio dei libri, ma deve partecipare egli stesso a quella vita tecnica, alla quale sono destinati $\mathrm{i}$ suoi allievi. Conscio della importanza di un tale principio il professor Morra si mantenne sempre in contatto colla tecnica, pur non prendendo parte attiva alla vita pratica industriale, dalla quale lo allontanavano $l^{\prime}$ indole dei suoi studii e lo stesso suo carattere. Per i medesimi motivi, egli non si dedicó con intensità alla professione di ingegnere; ciò nondi- 
meno peró, per la fama di speciale competenza che si era venuto acquistando, fu assai spesso chiamato da municipi, da amministrazioni pubbliche, da privati, ad esaminare progetti e ad eseguire collaudi di grandiosi impianti per il riscaldamento e la ventilazione di teatri, ospedali, scuole, stabilimenti industriali, ed altri importanti edificii.

Per i suoi meriti speciali fu spesso chiamato a far parte di commissioni esaminatrici in istituti rarii, ed in concorsi a posti di insegnante di fisica e chimica in scuole militari e professionali.

Fu membro prima e poi presidente della commissione peritale incaricata di giudicare sopra i ricorsi contro l'applicazione della tassa di consumo del gas e della luce elettrica: fu membro attivo della Sucietà di Igiene.

Collaborò con Galileo Ferraris nel Comilato organizzature e poi nella Giuria della Esposizione internazionale di elettricità di Torino nel 1884: anche maggiore attivita dedico alla Esposizione del 1898, l' esito della quale la morte del Ferraris rendeva assai dubbio.

A varie istituzioni di beneficenza presto pure l'opera sua diligente e disinteressata.

La preparazione delle molte lezioni che egli doveva svolgere, lo studio accurato, che, come giá ricordai, andava man mano facendo sulle varie pubblicazioni scientifiche e tecniche, poco tempo gli lasciavano disponibile per dedicarsi a ricerche originali. Non è questa peró la sola nè la principale ragione del fatto che l'opera sua scientifica non sia stata cosi feconda come dall'ingegno suo e dalla sua coltura si poteva attendere: intimamente modesto non fu forse lui stesso conscio del suo valore: educato ad un alto concetto della scienza era condotto a giudicare privo di interesse il far conoscere i risultati di quelle ricerche e di quegli studii ai quali egli pur con tanto amore si dedicava nel suo piccolo laboratorio: questi sentimenti in lui naturali erano resi anche più vivi dall'essersi trovato per tanto tempo a contatto di un genio 
sommo creatore di cose meravigliose. Per questi sentimenti egli ebbe una grande riluttanza allo scrivere e solo vi si decideva quando proprio non poteva declinare l'invito che enti ed associazioni gli venivano facendo: non rimane quindi di lui opera veramente originale; $\mathrm{i}$ suoi scritti sono piuttosto lavori di compilazione, nei quali però l'argomento è esaminato in ogni sua parte con particolare competenza ed esposto con frase precisa ed elegante.

In una memoria giovanile sopra le - Tensioni in un prisma - presentata nel 1877 come tesi per la laurea di ingegnere civile, il Morra studia le deformazioni e le tensioni o pressioni in un prisma sollecitato da una forza parallela al suo asse, nel caso in cui il solido non sia omogeneo, e quindi il coefficiente di elasticita abbia valori diversi da punto a punto.

Nel 1895 la direzione della Enciclopedia delle Arti e delle Industrie affidò al prof. Morra l' incarico di svolgere il capitolo relativo al - Riscaldamento dei locali di abitazione. - In quest'opera l'Autore descrive dapprima i varii sistemi di riscaldamento ed i relativi accessorii, corredando l' esposizione di abbondanti disegni e di schemi d'impianto; in seguito tratta per i varii sistemi dei procedimenti di calcolo, avendo cura, senza abbandonare il rigore scientifico, di semplificare la trattazione per modo da stabilire nel modo più facile le relazioni che servono ai casi della pratica; la trattazione teorica è completata con abbondanti citazioni di risultati sperimentali : quest' upera è in sostanza informata a quegli stessi concetti fondamentali che erano di guida al professore nello svolgimento del corso di fisica tecnica.

Alla morte di Galileo Ferraris la Società degli Ingegneri ed Architetti di Torino volle che fosse lui, il compagno fedele ed affezionato, a dettarne la commemorazione: in questa, pur tra la commozione vivissima di dover parlare di chi gli era tanto caro, il Moria analizza con acume critico le varie memorie e pubblicazioni del Ferraris, ponendo in rilievo l'alta importanza dell'opera sua di scienziato e di professore: come egli aveva fatto nella vita ne segue in questo scritto lo sviluppo del pensiero scientifico, ponendo in evidenza il coordinamento 
logico, col quale la mente del sommo scienziato fu condotta alle sue più importanti scoperte.

Incaricato dalla Presidenza del R. Museo Industriale di leggere il discorso inaugurale nella solenne riapertura degli studii in quest'anno, che egli purtroppo non doveva condurre a compimento, il prof. Morra scelse come tema - Le ricerche scientifiche ed $\mathrm{i}$ problemi di ingegneria. - In questo discorso, stabilita anzitutto la necessità che agli ingegneri si dia una profonda, non vasta e superficiale, ma salda e veramente efficace coltura matematica, osserva che la scienza del$l$ ' ingegnere non è di puro raziocinio, che essa è essenzialmente fisica, quindi di osservazione e di esperienza: con numerosi esempi dimostra essere impossibile separare la teoria dalla pratica, le esperienze dello scienziato dalle applicazioni che esse ricevono nella tecnica industriale, ponendo in rilievo la necessità che nei corsi di ingegneria tutte le materie di insegnamento siano corredate di adatti laboratorii, nei quali la mente degli allievi sia educata alla osservazione costante, per cui assurgendo dal complesso dei fatti al complesso delle leggi, trovi la via alle logiche applicazioni delle leggi stesse alla soluzione dei problemi tecnici. L'Autore è cosi condotto a concludere facendo voti che vengano aumentati sempre meglio agli studiosi coi laboratorii i mezzi di investigazione in quell' Istituto, allo sviluppo del quale egli aveva dedicata tanta parte della sua attività.

In questo voto il Morra voleva mirare principalmente al Laboratorio di Fisica Tecnica che prossimamente doveva venire riordinato in più aclatta sede nei nuovi locali, ricevendo quegli ampliamenti che già ad altri gabinetti avera saputo dare il R. Museo; l'organizzazione di questo laboratorio già da parecchi anni era studiata dal Morra, nel concetto del quale il laboratorio doveva servire non solo alle ricerche degli insegnanti, ed alle analisi svariate che l'Istituto compie per incarico di pubbliche e private amministrazioni, ma ancora ad una ben coordinata serie di esperimenti, che anche nel corso di fisica tecnica egli divisava di far eseguire dagli allievi ingegneri. 
Purtroppo questo lungo studio doveva essere senza frutto, egli non potè neppure iniziare ciò che con tanto annore avera studiato.

Ho già ricordato il carattere integro, l'animo dolce e mite del prof. Morra, e la eccessiva sua modestia che in ogni circostanza lo faceva ritroso a porre in evidenza sè stesso e il suo sapere: anche nelle conversazioni amichevoli non fece mai sfoggio della sua scienza; solo cogli intimi si lasciava portare a discussioni scientifiche e tecniche, e allora egli sapeva sviscerare a fondo la questione, richiamare i precedenti scientifici di essa, e con ragionamento stringato concludere ad un giudizio alto e sereno.

Per le doti del suo cuore e della sua mente il prof. Morra ebbe fra scienziati e tecnici salde amicizie fatte di stima e di affetto, delle quali grandemente si allietó; non fu però da tutti stimato al suo giusto valore, e non gli furono risparmiati gravi dolori e delusioni, che certamente ebbero grande influenza sulla malattia che ne travagliò gli ultimi anni e ne spezzò prematuramente l'esistenża.

Sulla tomba di Pietro Paolo Morra, se pure per la scienza possiamo rimpiangere che del suo non comune ingegno, della sua vasta coltura non rimanga opera più estesa e poderosa, dobbiamo inchinarci reverenti, perchè egli seppe acquistare due grandi titoli alla nostra ammirazione: la devozione fedele della quale costantemente circondò Galileo Ferraris, e l'amore intenso che egli portò alla scienza e che seppe infondere in una schiera di giovani, i quáli ora nei varii gradi delle carriere militari, scientifiche e tecniche si onorano di averlo avuto a Maestro.

Prof. L. Ferraris. 